\title{
Contrast echocardiography for left ventricular opacification
}

\author{
J Timperley, A R J Mitchell, H Becher
}

Heart 2003;89:1394-1397

$\mathrm{E}$ chocardiography has been criticised of having only moderate reproducibility and of having a low sensitivity of changes in left ventricular ejection fraction (LVEF). Inadequate endocardial visualisation during echocardiography occurs in up to $20 \%$ of cases. Although image quality has been improved with the introduction of harmonic imaging, ${ }^{1}{ }^{2}$ there are still a number of studies remaining of inadequate quality, and it is here that the use of contrast agents comes into its own. ${ }^{3}$ A number of studies have now been performed looking at the effect of contrast on assessment of cardiac regional and global function. This paper reviews the current and future clinical use of ultrasound contrast agents for assessment of left ventricular function.

\section{DEVELOPMENT OF CONTRAST AGENTS AND IMAGING TECHNIQUES}

The first licensed contrast agent in the UK was Levovist (Schering, Germany) and has now been followed by Optison (Amersham, UK) and Sonovue (Bracco, Italy) which are licensed for left ventricular opacification. Although infusions are preferential for assessment of myocardial perfusion, bolus injections of agents may be satisfactory for left ventricular opacification in many cases. In tandem with the development of contrast agents have been advances in imaging modalities. The first contrast specific modalities were harmonic imaging and harmonic power Doppler, which both use a relatively high transmit power leading to bubble destruction. Optimal imaging therefore requires triggered imaging. Second harmonic imaging enhances contrast effect compared to fundamental imaging and has been used for border definition using real-time imaging. ${ }^{4}$ However, it also leads to bubble destruction and artefacts. Latest developments such as power modulation and power pulse inversion, which are low power techniques and non-destructive, allow for real-time imaging without the limitations of harmonic imaging and with less contrast. As tissue returns are not displayed, unlike with high powered techniques, they are ideal for accurately delineating the left ventricular borders.

\section{CLINICAL APPLICATIONS}

\section{Left ventricular volume assessment}

Accurate assessment of left ventricular volumes requires adequate endocardial border delineation. Despite harmonic imaging, endocardial visualisation may still be inadequate; in one study this technique alone was insufficient to allow accurate determination of LVEF in $38 \%$ of difficult-to-image patients. ${ }^{2}$ In a study by Hundley and colleagues, ${ }^{3}$ volumetric assessment by echocardiography using Optison was compared to magnetic resonance imaging (MRI). They found correlation coefficients between echocardiographic and MRI techniques for end diastolic volume, end systolic volume, and ejection fraction $>0.92$. Following contrast injections, the 95\% confidence intervals for all three parameters were significantly smaller. However, it is important to remember that even following contrast injection, two dimensional (2D) echocardiographic derived volume data are dependent on accurate orientation of the scanning plane; foreshortening of the left ventricular cavity will lead to significant underestimation of volumes.

\section{Left ventricular thrombus}

Currently transthoracic echocardiography is the standard diagnostic procedure for the diagnosis of left ventricular thrombus and has been reported to have a sensitivity and specificity of $95 \%$ and $86 \%$, respectively. ${ }^{5}$ However, between $15-46 \%$ of echocardiograms are suboptimal in terms of quality for the identification of thrombus. ${ }^{67}$ Native imaging suffers from near field artefacts, where apical left ventricular thrombi are usually located in the apical views. The use of contrast has been shown to improve the diagnostic yield in patients with suboptimal native images, allowing $90 \%$ of initially non-diagnostic images to become diagnostic (fig 1).

\section{Stress echocardiography and regional assessment}

The assessment of regional wall motion at rest is important not just as the prelude to stress echocardiography; the presence of resting wall motion abnormalities has prognostic importance following myocardial infarction. Figure 2 demonstrates the extent of endocardial definition that contrast can provide. Stress echocardiography is recognised to be one of the most demanding techniques to learn in echocardiography but has been shown to have a high sensitivity and specificity for coronary heart disease. ${ }^{8}$ However, it is currently an entirely subjective assessment of regional wall motion and is dependent on the quality of the images recorded. Previous studies have shown that good endocardial visualisation is required for reliable assessment of regional wall motion. Images are worse during stress because of cardiac movement and also hyperventilation. In one study looking at interinstitutional observer agreement during dobutamine stress echocardiography, agreement of the presence of an abnormality on stress echocardiography was $73 \%$ for all studies but $100 \%$ when considering only images with the highest image quality and $43 \%$ for the lowest quality. ${ }^{9}$ Clinical studies have shown the benefit of contrast in improving image quality, percentage of wall segments visualised, and confidence of interpretation of regional function both at rest and at peak stress. $^{10}$

\section{Automatic boundary detection for volume and regional wall assessment}

The standard echocardiographic technique for left ventricular volume assessment is manual tracing of endocardial borders and volume calculation using Simpson's method. However, this is time consuming and tedious, although the use of

\footnotetext{
Abbreviations: $2 \mathrm{D}$, two dimensional; $3 \mathrm{D}$, three dimensional; $\mathrm{AQ}$, acoustic quantification; LVEF, left ventricular ejection fraction; MRI, magnetic resonance imaging
} 


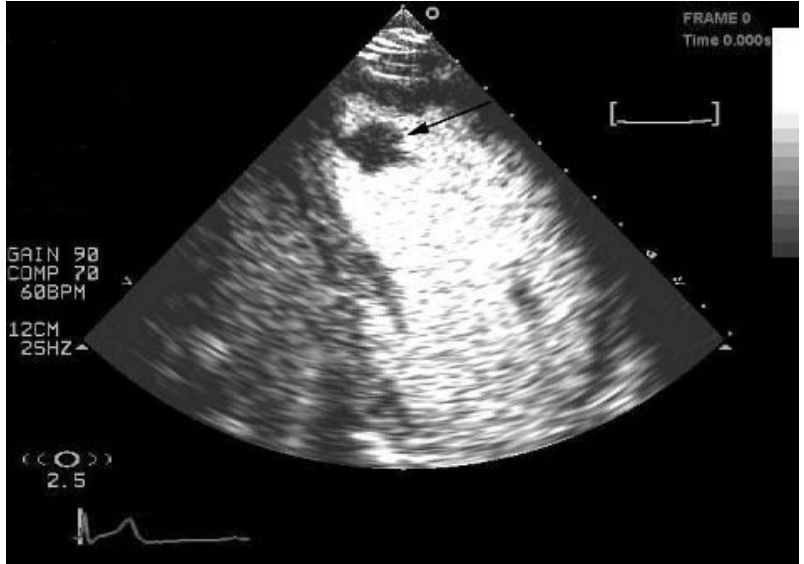

Figure 1 Apical left ventricular thrombus (arrow) on two chamber apical view demarcated by contrast.

contrast does improve the results compared to native harmonic imaging. ${ }^{2}$ Automatic boundary detection obviating the need for manual tracing has been a goal for a number of years, but with native images numerous techniques have failed to track adequately. With contrast and low power techniques we have the ideal conditions for these automatic boundary detection algorithms to work. Acoustic quantifica- tion (AQ) is one technique for boundary detection. This was found to be more accurate at left ventricular volume assessment with contrast compared to AQ on native images. ${ }^{11}$ Another recent study using a different boundary detection technique has shown similar results to manual tracing in 51 patients undergoing contrast echocardiography using power harmonic imaging and bolus injections of Levovist, ${ }^{12}$ with good correlation to volumes from radionuclear angiography. Initial results from an automatic boundary detection algorithm currently in use in our centre have shown the ability to improve interobserver and intraobserver variability in the assessment left ventricular volumes compared to hand tracing (fig 3). The same software can also provide colour coded information on regional motion (fig 4).

\section{Three dimensional echocardiography}

The obvious benefits of the use of contrast in 2D echocardiography will soon be extended to three dimensional (3D) echocardiography. The precise geometric data from 3D echocardiography based upon the attainment of multiple $2 \mathrm{D}$ images and interpolation into a 3D dataset allows for more detailed studies of valvar and congenital heart disease. Initial studies used free-hand imaging, whereby a standard 2D probe was fanned across the heart and 3D reconstruction was performed off-line. The advent of real-time 3D echocardiography will reduce the limitations seen with 2D echocardiography, in particular the inability of 2D echocardiography to display complex left ventricular geometry such

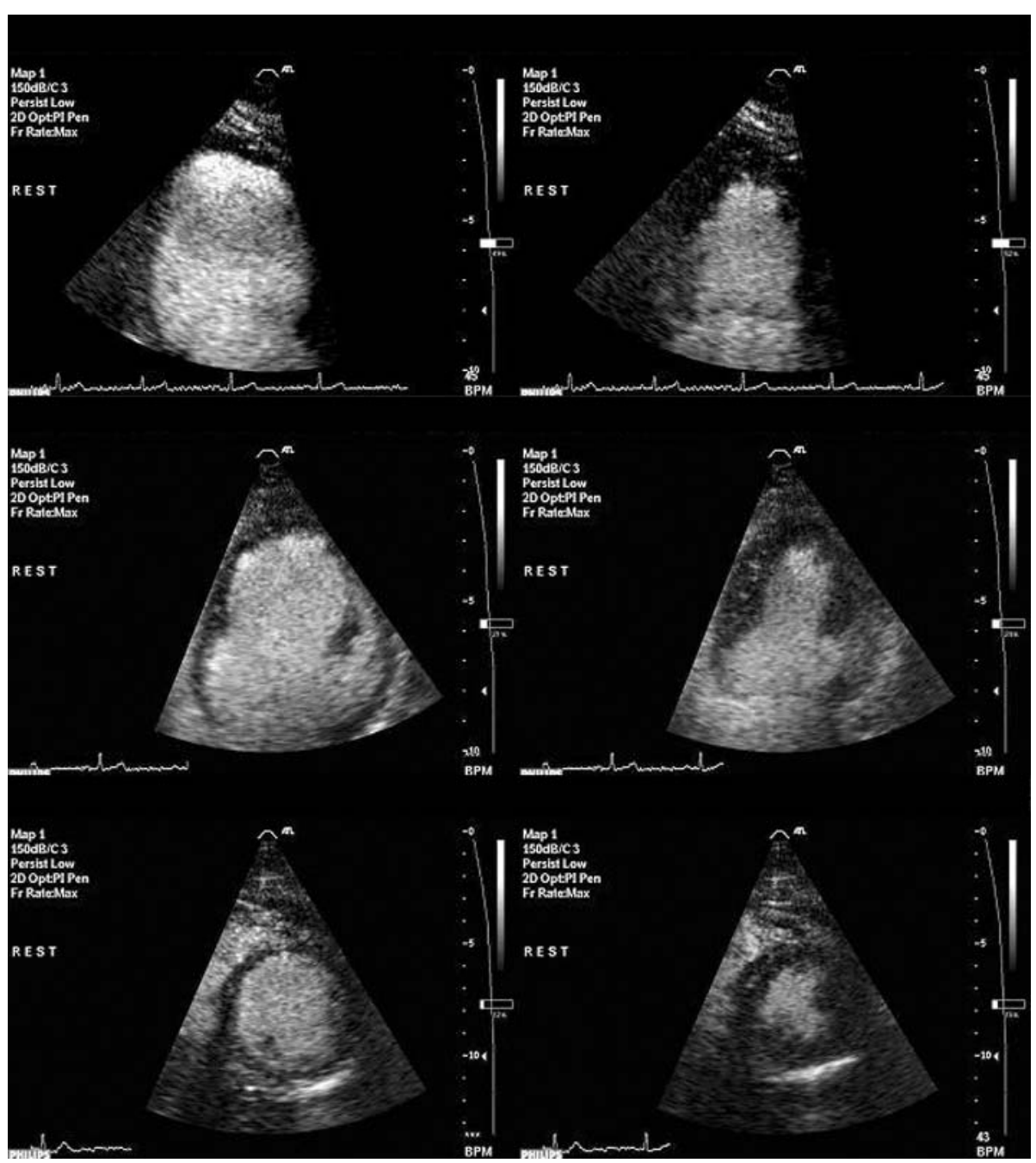

Figure 2 End diastolic and end systolic frames in two chamber, four chamber, and parasternal short axis views. 

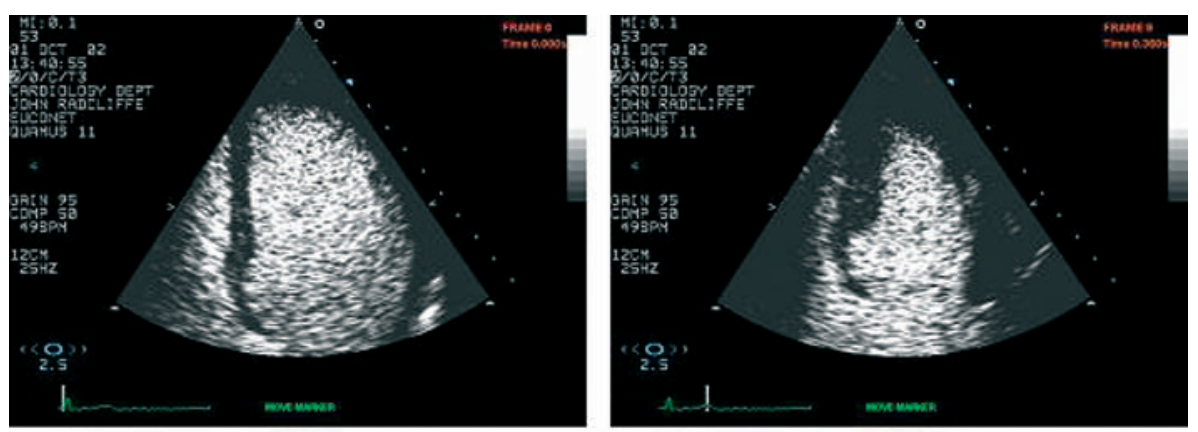

Figure 3 End diastolic and end systolic views of contrast view, segmented view as derived by the computed algorithm, and borders superimposed on the contrast image (Quamus, Mirada Solutions, UK).
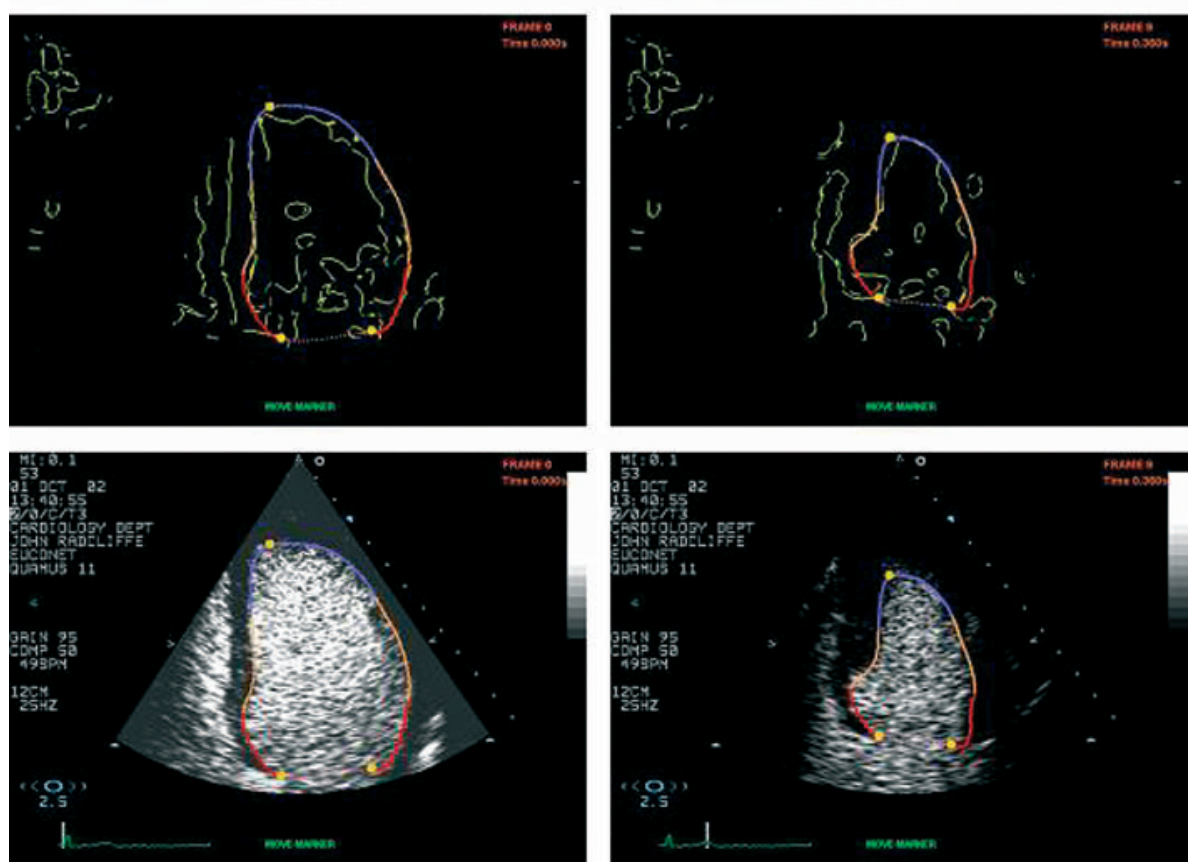

as with aneurysms. Already, 3D volumetric analysis has been shown to be more accurate than 2D studies with reduced inter-operator variability, ${ }^{13}$ and this is likely to be due to the assumption of symmetrical left ventricular geometry with $2 \mathrm{D}$ techniques. ${ }^{14}$

However, 3D imaging still has limitations relating to image quality and also the time consuming and cumbersome volume analysis requiring hand tracing of multiple short axis left ventricular slices. 3D echocardiography is therefore the ideal imaging modality to use synergistically with contrast agents. The accurate measurements provided by $3 \mathrm{D}$ techniques, coupled with the enhanced endocardial border identification provided by contrast agents, lead to a quick and effective method of assessment of left ventricular function. The opportunity to perform automatic analysis of a 3D dataset is now available.

\section{CONCLUSION}

Contrast left ventricular opacification improves the proportion of segments visualised at rest and with stress. It also improves volumetric assessment compared to native images. The improvement in boundary delineation has allowed for the development of a number of different techniques for automatic boundary detection, which should in the future shorten the time for volumetric assessment and may lead to the holy grail of regional and global assessment-automatic and quantitative results. The future potential of combined 3D imaging with contrast has the potential of similar 3D dataset as from MRI, but with a shorted acquisition time and studies to assess this are currently ongoing.

\section{ACKNOWLEDGEMENTS}

M Mulet and B Javier for work with the Quamus Software.

\section{Authors' affiliations}

J Timperley, A R J Mitchell, H Becher, John Raddliffe Hospital, Oxford, UK

Correspondence to: Dr Harald Becher, Department of Cardiology, John Radcliffe Hospital, Oxford OX3 9DU, UK; harald.becher@orh.nhs.uk

\section{REFERENCES}

1 Senior R, Soman P, Khattar RS, et al. Improved endocardial visualization with second harmonic imaging compared with fundamental two-dimensional echocardiographic imaging. Am Heart J 1999;138:163-8.

2 Yu EH, Sloggett CE, Iwanochko RM, et al. Feasibility and accuracy of left ventricular volumes and ejection fraction determination by fundamental, tissue harmonic, and intravenous contrast imaging in difficult-to-image patients. J Am Soc Echocardiogr 2000;13:216-24.

3 Hundley WG, Kizilbash AM, Afridi I, et al. Administration of an intravenous perfluorocarbon contrast agent improves echocardiographic determination of left ventricular volumes and ejection fraction: comparison with cine magnetic resonance imaging. J Am Coll Cardiol 1998;32:1426-32.

4 Allen MR, Pellikka PA, Villarraga HR, et al. Harmonic imaging: echocardiographic enhanced contrast intensity and duration. Int J Card Imaging 1999:15:215-20.

5 Stratton JR, Lighty GW, Jr, Pearlman AS, et al. Detection of left ventricular thrombus by two-dimensional echocardiography: sensitivity, specificity, and causes of uncertainty. Circulation 1982;66:156-66.

6 Shaw L. Impact of contrast echocardiography on diagnostic algorithms: pharmacoeconomic implications. Clin Cardiol 1997;20:139-48.

7 Thanigaraj S, Schechtman KB, Perez JE. Improved echocardiographic delineation of left ventricular thrombus with the use of intravenous secondgeneration contrast image enhancement. J Am Soc Echocardiogr 1999;12:1022-6. 


\begin{tabular}{|c|}
\hline $\mathrm{BS}$ \\
$\mathrm{MS}$ \\
$\mathrm{AS}$ \\
$\mathrm{AL}$ \\
$\mathrm{ML}$ \\
$\mathrm{BL}$ \\
\hline
\end{tabular}
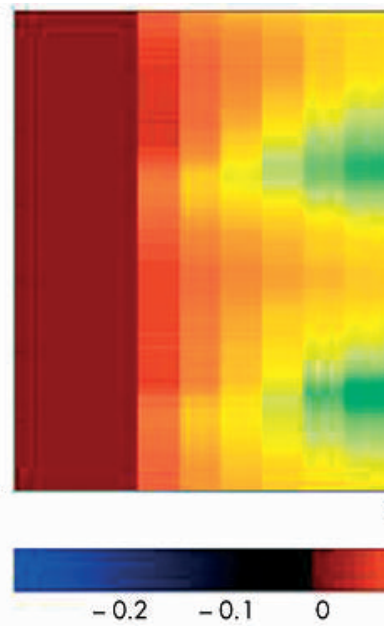

0.2

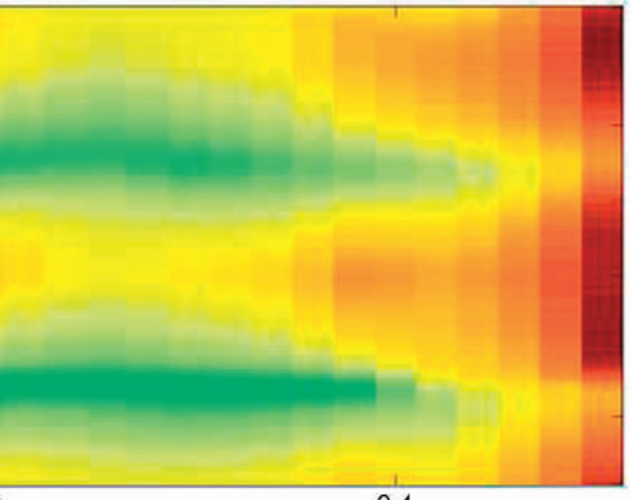

0.4

Time in seconds
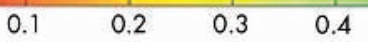

0.5
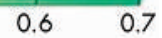

\begin{tabular}{|l|}
\hline $\mathrm{BS}$ \\
$\mathrm{MS}$ \\
$\mathrm{AS}$ \\
$\mathrm{AL}$ \\
$\mathrm{BL}$
\end{tabular}

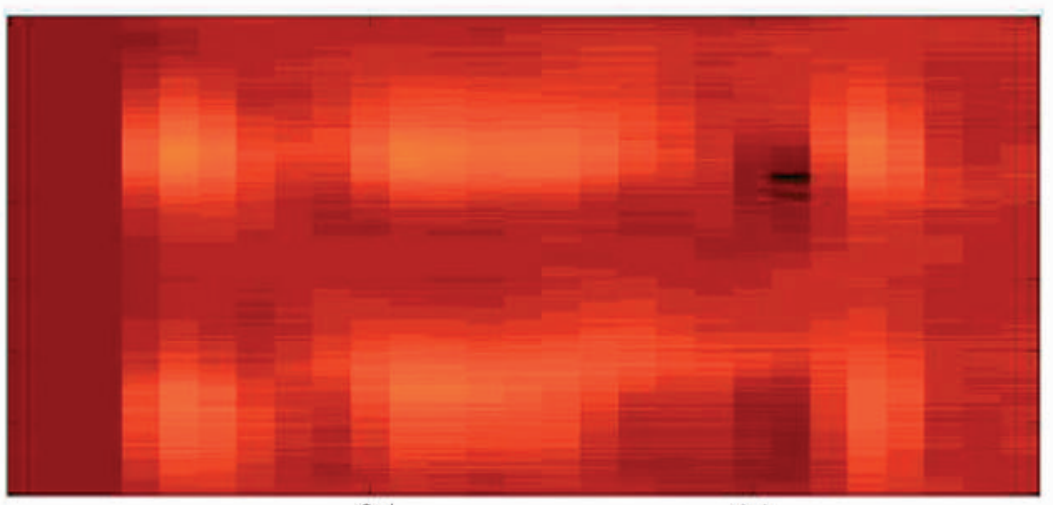

0.4
0.8

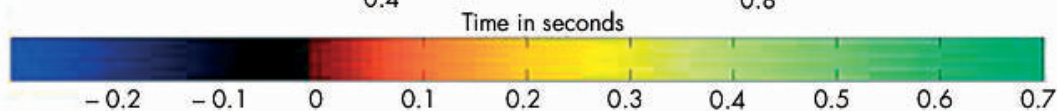

Figure 4 Colour coded images of regional function in normal and abnormal studies using automatic boundary detection. Colour scale represents displacement of endocardium compared to a central geometric template (blue outwards that is, dyskinetic, green peak inwards) over a cardiac cycle in a four chamber image. Upper image normal, lower globally impaired with akinetic apex and hypokinetic basal and mid segments. BS, MS, AS = basal, mid, apical septum, respectively; $A L, M L, B L$ = apical, mid, basal lateral wall, respectively.
8 Pellikka PA, Roger VL, Oh JK, et al. Stress echocardiography. Part II. Dobutamine stress echocardiography: techniques, implementation, clinical applications, and correlations. Mayo Clin Proc 1995;70:16-27.

9 Hoffmann R, Lethen H, Marwick T, et al. Analysis of interinstitutional observer agreement in interpretation of dobutamine stress echocardiograms. J Am Coll Cardiol 1996;27:330-6.

10 Rainbird AJ, Mulvagh SL, Oh JK, et al. Contrast dobutamine stress echocardiography: clinical practice assessment in 300 consecutive patients. J Am Soc Echocardiogr 2001:14:378-85.

11 Spencer KT, Bednarz J, Mor-Avi V, et al. Automated endocardial border detection and evaluation of left ventricular function from contrast-enhanced images using modified acoustic quantification. J Am Soc Echocardiogr 2002;15:777-81.

12 Yu EHC, Skyba DM, Sloggett CE, et al. Determination of left ventricular ejection fraction using intravenous contrast and a semiautomated border detection algorithm. J Am Soc Echocardiogr 2003:16:22-8.

13 Takuma S, Ota T, Muro T, et al. Assessment of left ventricular function by realtime 3-dimensional echocardiography compared with conventional noninvasive methods. J Am Soc Echocardiogr 2001;14:275-84.

14 Tortoledo FA, Quinones MA, Fernandez GC et al. Quantification of leff ventricular volumes by two-dimensional echocardiography: a simplified and accurate approach. Circulation 1983;67:579-84. 